\title{
La chair ingénue : manipuler l'enfant et son image durant la Grande Guerre. L'exemple particulier des atrocités allemandes en France.
}

Thaïs BIHOUR, Université Paris 1 - Panthéon Sorbonne, HiCSA

\section{Introduction}

Pour les sociétés engagées dans la Grande Guerre, les enfants constituent un enjeu vital pour un futur idéal. Dans un État dont on prétend qu'il sortira plus fort de l'épreuve, ils doivent eux-mêmes jouer un rôle tout au long des cinquante-deux mois du conflit et témoigner de leur haine de l'ennemi en tant que bons patriotes. (Prévost-Bault 134)

En ce qui concerne la Grande Guerre, dire que le degré d'engagement de la jeunesse fut élevé serait un euphémisme : aucun conflit jusque-là n'avait utilisé à ce point l'enfant et son fort potentiel symbolique. En effet, la Première Guerre mondiale est caractérisée par son aspect de guerre totale, touchant de manière presque indifférenciée les combattants et les civils ; les limites entre les zones de combats et celles de l'arrière sont parfois floues et les bombardements font beaucoup de victimes hors du champ de bataille. Par là même, elle nécessite l'investissement de toute la population, enfants y compris. Tous les moyens sont alors mis en œuvre pour faire intégrer à la nouvelle génération cette haine viscérale de l'ennemi, et c'est ainsi que l'Ecole, les parutions illustrées pour la jeunesse ou encore certains jouets apparaissent comme des instruments patriotiques redoutables.

Mais ce qui semble avant tout justifier cette forte mobilisation des enfants et tous les efforts qui leur sont demandés, c'est que la France ne cessera jamais de crier son engagement au nom du Droit : il est nécessaire de prouver aux combattants ainsi qu'à l'opinion, qu'il ne s'agit pas d'une guerre offensive amorcée par la France, mais bien d'une guerre défensive en réaction aux ambitions expansionnistes de l'Allemagne. Protéger sa Patrie ainsi que la jeune génération devient alors l'une des grandes justifications de la guerre, constituant d'ailleurs l'un des piliers les plus solides du consentement collectif.

Le motif particulier des atrocités allemandes ${ }^{1}$ que nous avons choisi de mettre ici en valeur, représente une thématique abondamment répandue dans la presse française. Mais que cache réellement l'expression d'atrocités allemandes à l'heure du grand conflit mondial ?

A partir du 4 août 1914, l'armée allemande envahit la Belgique - alors neutre à ce moment-là - et le Nord-Est de la France. Confrontés à une forte résistance de la population qu'ils n'avaient pas envisagée, les soldats allemands réagissent avec force et véhémence afin d'endiguer toute protestation et parer à d'éventuelles attaques de francs-tireurs. Commence 
alors une vague d'exactions d'une violence extrême, où les témoignages d'incendies, pillages, destruction du patrimoine, viols, exécutions de masse et tortures envers les femmes, les personnes âgées et les enfants, s'étalent en première page des journaux et s'accompagnent d'une production iconographique pléthorique. Si l'on pourrait s'attendre à ce que les enfants soient avant tout utilisés pour leur propension à incarner le motif même de l'innocence et de la fragilité, nous sommes alors bien loin de la réalité des années 1914-1918 : certes l'iconographie victimaire est présente, mais l'exaltation héroïque et patriotique la surpasse véritablement, installant par là même un discours insidieux, culpabilisant et fortement restrictif $^{2}$ pour cette jeunesse confrontée à la guerre. Dès lors, par quels procédés l'enfant estil amené à intégrer des concepts patriotiques et guerriers, normalement éloignés des préoccupations juvéniles qui sont les siennes? Et comment les diverses parutions illustrées pour la jeunesse parviennent-elles à investir l'enfant d'un rôle normalement dévolu aux adultes?

Dans un premier temps, il conviendra d'aborder le rôle de l'Ecole et son importance dans la diffusion de l'image de l'ennemi pour comprendre le climat guerrier dans lequel étaient éduqués les écoliers français, un climat fortement antigermanique, bien sûr, qui les rend d'autant plus perméables aux idées distillées dans les journaux pour enfants une fois rentrés chez eux. Puis, à l'aide de quelques exemples choisis, nous observerons comment ceux-ci parviennent à remplir deux objectifs primordiaux pour la valorisation $\mathrm{du}$ combat français : investir l'enfant du rôle de petit soldat en herbe et montrer l'Allemand comme un être malfaisant, cruel par essence mais, surtout, dénué de toute intelligence.

\section{Un climat propice à distiller la haine : le rôle de l'École dans la diffusion de l'Allemand comme ennemi héréditaire}

En 1918, est publié le roman La chair innocente, l'enfant du viol boche, dans lequel nous est racontée l'histoire d'une femme enceinte suite à un viol allemand, et qui refuse contre l'avis de sa famille, d'abandonner son enfant. Innocence, tel est bien le mot qu'il faut retenir ici, car les enfants ont une place et un rôle particuliers à jouer dans le déroulement de la Grande Guerre. En effet, les adultes se chargent de leur assigner un double rôle qui semble largement les dépasser: à la fois victimes mais aussi promesse d'un renouvellement générationnel, ils ne peuvent échapper à un discours culpabilisant qui se referme sur eux. A ce titre, les difficultés endurées par les petits Français sont parfaitement bien illustrées par les propos de Françoise Dolto, toute jeune au moment du conflit : 
Et naturellement, j'étais très ennuyée de ça, je passais mon temps à faire du tricot, je ne pouvais même plus jouer parce qu'on me culpabilisait, ils l'attendaient soi-disant. Il y avait un pauvre poilu dans les tranchées qui attendait mon cache-nez et qui mourrait de froid si je ne finissais pas son cache-nez. Et je ne me rendais pas compte que ça amusait les gens, de me voir toute pénétrée de la valeur importante de ce qui était une corvée pour moi, à la longue. Je ne sais pourquoi, c'était un peu sadique d'ailleurs vis-à-vis d'un enfant, et j'ai compris après que, très souvent, quand les gens venaient, ils venaient me regarder comme ça, affairée à mon tricotin. (28)

Mais si les enfants ont été investis de rôles bien trop lourds pour eux, il faut avant tout comprendre par quels moyens ils ont été sensibilisés aux discours patriotiques. Dans $L a$ guerre des enfants. 1914-1918, Stéphane Audoin-Rouzeau revient précisément sur le rôle dont ces derniers ont été investis pendant la Première Guerre mondiale, et par quels moyens ils ont été sensibilisés aux discours patriotiques, notamment par le biais de l'Ecole, de l'Eglise ou encore de jeux dans lesquels les Allemands sont stigmatisés, caricaturés ou exhibés comme de véritables barbares. Parmi eux, l'exemple d'un puzzle constitué de cubes dont l'assemblage final révèle le dessin d'un enfant baignant dans son sang après avoir été abattu par un soldat allemand, est assez révélateur du discours ambiant. Il en va de même pour le plateau de jeux de l'Oie ${ }^{3}$ dessiné par l'illustrateur Guy Arnoux, dont chaque case contient une petite image satirique antigermanique.

De manière plus étonnante mais tout aussi caractéristique, le conte du Petit chaperon rouge, dont l'histoire a été adaptée au contexte guerrier par Charles Moreau-Vauthier et Guy Arnoux en 1917, agit dans un esprit similaire. Cet ouvrage destiné à un jeune lectorat est proprement exceptionnel, tant par la violence iconographique qu'il met en exergue, que par sa réécriture aux accents vindicatifs. Dans ces pages, le loup prend l'apparence d'un officier allemand: casque à pointe, long manteau et bottes de militaire noirs ainsi que sabre au fourreau, tous les symboles sont réunis pour que l'image soit rapidement assimilée. En face de l'animal-soldat, se tiennent à tour de rôle, une petite fille vêtue tout aussi symboliquement d'une robe tricolore et d'un petit chapeau qui ressemble à s'y méprendre à un bonnet phrygien - la France, ainsi qu'une autre enfant, quant à elle baptisée «Petite Belgique »; tandis que «Mère l'Europe » et «Grand'Mère la Paix » veillent sur leurs petites nations vaillantes. Toutes finiront d'ailleurs par vaincre et étriper littéralement le grand méchant «Loup Boche », lui arrachant la queue et les moustaches tout en l'assommant à grands coups de tisonnier. Cette violence, ce sont ici des enfants qui la commettent sous la bannière d'une justification héroïque : celle de la défense de leur Patrie. Mais si nous poussons l'analyse plus avant, on ne peut s'empêcher de songer aux nombreuses interprétations - notamment 
sexuelles - qui ont été faites de l'histoire du Petit chaperon rouge ; pensons ne serait-ce qu'un instant à La psychanalyse des contes de fées de Bruno Bettelheim et à la couleur rouge, d'abord, symbole des émotions violentes et particulièrement celles qui ont trait à la sexualité, au combat antagoniste de la tentation du plaisir face à la réalité, à l'acte symbolique de dévoration ou encore à la dualité incarnée par les personnages masculins, à la fois séducteur et dangereux pour le loup, mais aussi sauveur et altruiste pour le chasseur.

En ce sens, la réécriture de ce conte dans le contexte de la Grande Guerre pourrait être vu comme le miroir - enfantin - des violences sexuelles commises envers les femmes durant l'invasion, des viols et mutilations que les artistes français ont d'ailleurs représentés à outrance entre 1914 et 1916, esthétisant parfois leurs compositions jusqu'à dépeindre des victimes dans des positions lascives, offertes à leurs bourreaux (sur l'esthétisation des violences sexuelles durant la Grande Guerre, voir Bihour). Mais le support du conte s'adressant à un jeune public et ne permettant pas l'allusion sexuelle trop directe, le recours à la métaphore serait donc un moyen d'insinuer - consciemment ou non - les débats et enjeux patriotiques de l'époque dans l'esprit des enfants. Au fond, que le Petit chaperon rouge soit un reflet dissimulé de l'altérité des adultes ou plus simplement, un moyen détourné d'inciter à la stigmatisation de l'ennemi, il est au moins évident que la mise en scène du méchant Loup Boche attaquant Grand'Mère la Paix, ne laisse aucun doute sur l'impact qu'une telle iconographie pouvait avoir sur les enfants.

Mais la pédagogie de guerre appliquée aux enfants ne passe pas uniquement par le divertissement ou les belles images colorées : elle passe aussi par l'enseignement scolaire de la Morale et de l'Histoire. Le passé historique et guerrier de la France est un vecteur de choix pour susciter l'engouement patriotique et il n'est pas rare que des illustrés pour enfants produisent une grande quantité de récits exaltant le glorieux passé de la Nation. Mais qu'apprend l'Ecole à ses petits élèves ? Elle leur apprend notamment que :

Si la France est, de tous les pays, le plus aimable, c'est aussi qu'elle a, plus qu'aucun autre pays, un passé prestigieux. Les écoliers français sont bien heureux, car les raisons qu'ils ont de prendre parti pour leur pays sont indiscutablement fondées : aucun autre peuple n'a fait 1789. 1789, c'est le changement qui crée véritablement la Patrie [...] et donne ses assises au vrai sentiment patriotique, au «patriotisme selon la Révolution ». 1789 réalise donc l'identification de la France avec les valeurs les plus hautes. La France devient une personne morale. (Ozouf 7-8)

Bien sûr, il n’y a pas que la Révolution française qui soit évoquée au sein des manuels scolaires, mais cet évènement pose néanmoins les bases d'un sentiment patriotique très fort auquel les enfants doivent être particulièrement sensibles. Notre but ici n'est pas de retracer 
une histoire de la pédagogie française ou de paraphraser les travaux majeurs déjà existants sur le sujet des enfants dans la guerre, comme ceux d'Audoin-Rouzeau que nous citions précédemment. Ce que nous cherchons à percevoir, ce sont les fondements de cette haine antiallemande, afin de mesurer l'impact des représentations d'atrocités et mythes d'atrocités allemandes telles qu'elles seront publiées au sein des productions illustrées destinées à la jeunesse - dont nous parlerons plus loin.

Ainsi, l'enseignement distribué par le biais des manuels scolaires est-il si belliqueux qu'il en a l'air? Pas vraiment : il est plutôt insidieux. En effet, les auteurs tentent de parvenir à un compromis pas toujours facile à approcher cependant, la principale difficulté étant que l'on s'adresse à des enfants. Ces derniers ne sont pas toujours capables de saisir les subtilités de la notion de guerre juste et confondent parfois amour de la Patrie et exaltation guerrière ; vouloir montrer la guerre comme un procédé monstrueux tout en la justifiant à certains égards, voilà toute la complexité de la tâche. Mais contrairement à ce que l'on pourrait penser, ces manuels font très attention à ne pas présenter la guerre sous des traits biologiques : si les Allemands peuvent être vus comme nos ennemis héréditaires, ils ne sont pas pour autant des barbares par essence, contrairement à ce qu'affirment beaucoup de discours nationalistes durant la Grande Guerre. Comment comprendre ce discours à revers du climat ambiant ? Justement comme une réponse à la question de guerre juste, réponse qui permet en plus, de faire retomber en intégralité, les torts sur l'adversaire :

Cette perspective délibérément politique a un grand avantage, elle permet de faire le tri entre les mauvaises et les bonnes guerres, que tous les manuels esquissent. Car, si la guerre est toujours un mal, il y a des maux pires encore : la perte de la liberté et de la dignité [...] Dans le cas où elles seraient menacées, il faut faire la guerre, et on peut la faire sans participer au mal. Alors, l'odieux en retombe sur les barbares qui l'ont rendue inévitable. (Ozouf 18-19)

Mais les instituteurs ont aussi leur rôle à jouer au sein de la guerre : ils doivent à la fois poursuivre leur mission éducative afin de maintenir un bon niveau de scolarité dans le pays, tout en glorifiant les héros qui se battent pour la France, et donc pour rester dans l'esprit culpabilisant des discours de l'époque, pour les enfants. La plupart est plutôt réceptive aux discours et valeurs véhiculés par la propagande, tant est si bien que les cas où les instituteurs se révoltent contre ce bourrage de crâne et cette propagande anti-Boches sont trop rares pour être vraiment significatifs; dans l'ensemble et sur le long terme, l'Ecole va plutôt contribuer à renforcer les oppositions entre Français et Allemands: on stigmatise l'âme germanique sombre et féroce vis-à-vis du génie français et l'on oppose les termes de Kultur et Culture. 
Mais ce qui est significatif ici, c'est de constater que les enfants ont pu être à un moment donné de leur scolarité, confrontés à l'iconographie des atrocités allemandes dans les salles de classe. En effet, lors de l'année 1915, deux députés français du département du Gers, Messieurs Noulens et Gardey, écrivent au Ministre de l'Instruction Publique : «Tandis que la tragédie se déroule, nous voudrions voir les murs des salles de classe s'orner d'estampes vulgarisant les compositions de nos meilleurs artistes... Quel moyen plus sûr, en vérité, que cette saisissante évocation, pour montrer et graver dans les mémoires les excès de la force, les atrocités allemandes, les deuils et la ruine de la guerre [...] » (Revue pédagogique, n.p.).

La volonté de ces deux hommes ne reste pas sans suite et trouve même un écho considérable au sein des écoles. Ce qu'il faut bien comprendre, c'est que si les auteurs de manuels scolaires tentent, comme nous l'avons dit, de tempérer les ardeurs militaristes et nationalistes, les instituteurs agissent selon leurs propres initiatives, qui vont pour la plupart dans le sens du consentement collectif et de la propagande. Christian Peureux dans son travail de maîtrise sur «La propagande à l'école de 1914 à 1918 » explique que les maîtres d'écoles n'hésitent pas à disserter sur les atrocités allemandes et demandent parfois à certains enfants qui auraient vécu ces mêmes exactions, de raconter leur histoire devant leurs camarades ${ }^{4}$.

Mais ce contact des enfants avec les représentations d'exactions ne s'arrête pas aux portes des écoles. Lorsqu'ils sont dans leurs foyers, c'est aux parutions illustrées pour la jeunesse de prendre le relai. Ces revues destinées aux enfants, mêlant récits d'aventures historiques, petits romans de guerre, devinettes et iconographie guerrière se révèlent d'une grande violence, dont nous allons à présent constater l'étendue.

\section{Insinuer la haine de l'ennemi dès le plus jeune âge : l'impact de la presse enfantine illustrée}

Pour cette partie concernant les illustrations de la presse enfantine, nous nous appuierons principalement sur l'exemple de deux journaux : Les trois couleurs ${ }^{5}$, dont nous avons compulsé les années 1914, 1915, 1916 et 1917 ; et La jeune France ${ }^{6}$, pour les années 1915, 1916, 1917 et 1918. Nous tenterons à travers eux de percevoir l'évolution des représentations d'atrocités allemandes et l'impact que de telles images pouvaient avoir sur les enfants. Nous évoquerons aussi le cas spécifique des aventures de Bécassine, ce personnage étant situé dans un entre-deux qui consistait à légitimer la guerre auprès des enfants par la stigmatisation de la barbarie allemande, sans pour autant céder aux excès propagandistes et nationalistes. 
S'il n'est pas véritablement surprenant de constater un déferlement de représentations d'atrocités allemandes dans la presse destinée à un public d'adultes, il peut sembler plus étonnant de voir un tel sujet affiché dans des productions destinées à la jeunesse, d'autant plus que la violence des images et des propos qui leurs sont associés peuvent nous heurter de manière rétrospective. Ces journaux, en effet, n'hésitent pas à montrer des cadavres, des combats ou des exactions commises envers de très jeunes bambins ${ }^{7}$. Ces productions vont jusqu'à légitimer et exalter le courage des enfants piégeant et tuant des soldats allemands : là encore, le discours patriotique fonctionne à plein et les enfants apparaissent comme des cibles privilégiées, car très influençables ; cela, « Tous les camps l'ont compris. [...] Enfant victime ou enfant mobilisé : les enfants demeurent de la chair à canon» (Gervereau 105).

Concernant l'évolution du motif des atrocités allemandes au sein de la littérature de jeunesse, il est intéressant de constater que ce dernier suit une courbe descendante année après année. Le phénomène se constate d'ailleurs parallèlement dans les journaux destinés aux adultes, alors que les atrocités allemandes et leurs victimes commencent dès 1916 à être volontairement occultées au profit de l'effort de guerre. Ainsi, pour La jeune France, l'année 1915 compte vingt-deux représentations en lien avec le sujet qui nous occupe. Mais de manière symptomatique, le nombre descend brutalement à cinq pour l'année 1916. Quant aux années 1917 et 1918, elles ne contiennent qu'une illustration chacune sur notre sujet. Pour Les trois couleurs, le constat est exactement le même: nous pouvons compter treize illustrations pour l'année 1915 et seulement une seule en 1916, où l'on voit un nouveau-né jouer avec un hochet-obus.

Cet abandon progressif du motif, nous l'avons esquissé, trouve son explication dans les impératifs propagandistes. En effet, au fur et à mesure que la guerre avance, un climat de tensions se créé au sein de la population française, jusqu'à mener à un rejet massif de ces victimes que l'on considérait jusqu'alors comme des martyres, et qui ne deviennent aux yeux des gens que des bouches encombrantes à nourrir. Alors que les soldats sont mutilés et décimés sur le champ de bataille, ces femmes et enfants affaiblis semblent inutiles à l'effort de guerre : ils sont alors stigmatisés et évincés peu à peu du débat public et des journaux illustrés (voir Becker). Cependant, si l'abandon du motif s'avère symétrique tant dans la presse enfantine qu'adulte, force est de constater que son utilisation au début de la guerre ne vise pas exactement les mêmes objectifs.

Ce qui revient le plus souvent au sein des textes accompagnant les illustrations, c'est donc cet esprit très belligérant et vindicatif qui présente les enfants comme de petits soldats en herbe. D'ailleurs, des titres tels que «La valeur n'attend pas le nombre des années ${ }^{8}$ ou 
«Emile Desjardin, héro de quatorze ans ${ }^{9}$ donnés aux petites vignettes et bandes dessinées en sont tout à fait représentatifs. Cela diverge véritablement des objectifs ciblés par la presse destinée aux adultes, et ce pour plusieurs raisons, la première étant que jamais nous ne trouverons de représentations de violences sexuelles placées sous le regard d'un jeune public; il y a là un interdit moral qui ne réside pas tant dans la monstration de la violence elle-même que dans l'évocation de la sexualité, totalement prohibée. La seconde en est que, parmi toutes les représentations d'atrocités allemandes visant un public mûr, nous avons plutôt l'impression d'être face soit à des caricatures montrant l'Allemand comme un véritable barbare violeur de femmes, soit à des démonstrations de souffrances emplies de pathos. Quoi qu'il en soit, le mal est souvent déjà fait, et le ressenti face à ces images tient, semble-t-il, plutôt de l'horreur et de la pitié. Or, celles à destination des enfants les enjoignent pratiquement à prendre les armes ou à se battre au front, à élaborer des tactiques machiavéliques pour prendre des soldats allemands au piège ou à défendre leur mère contre les baïonnettes ennemies au péril de leur propre vie.

Les adversaires y sont montrés dévastateurs : ainsi en est-il de ces planches où le personnage de Bécassine tente de décharger un pistolet au beau milieu d'un salon. Dans sa maladresse, les coups partent et la pièce est saccagée. La marquise chez qui se trouve Bécassine, s'exclame alors en entrant : «C'est à croire que le Kronprinz et son état-major y ont passé » (Bécassine pendant la guerre, 46). Mais ce qui est particulièrement frappant, c'est l'empressement des illustrateurs à les désigner comme des êtres d'une grande stupidité, alors que les enfants sont ici héroïques et dotés d'une intelligence grandiose : il n'est pas rare que les Allemands se laissent berner par des enfants ${ }^{10}$ ou tombent malades après avoir bu du vin qu'un enfant aurait délibérément empoisonné ${ }^{11}$. Cette idée n'est pas uniquement véhiculée par la presse illustrée, puisque certains jeux d'adresse «proposent de tirer sur un 'boche' grotesque, craintif, qui a vite fait de lever les bras en criant 'Kamerad' » (Prévost-Bault 141). Mais il faut noter que parmi ces productions destinées à ce jeune public, toutes ne se sont pas autant soumises aux codes de la propagande. Ainsi en est-il du personnage de Bécassine.

Née le 2 février 1905, elle apparaît dans La semaine de Suzette, une publication destinée aux jeunes filles. Avec elle, il n'y a jamais de combats directs - que cela soit sur le champ de bataille ou à l'arrière - et encore moins de scènes explicites de meurtres. Chez Bécassine, il semble que les atrocités allemandes n'existent guère et les rares fois où des violences réelles sont évoquées, tels que les bombardements, elles ne sont jamais représentées, mais simplement suggérées. Nous pourrions penser qu'en prenant ce parti, les deux auteurs Joseph Pinchon et Cauméry - de son vrai nom Maurice Languereau - se placent 
totalement en marge du patriotisme ambiant de la Grande Guerre. Pourtant, même si nous avons souligné que les aventures de la petite Bretonne ne cédaient pas à la tentation propagandiste, elles n'en restent pas moins proches des soucis de leur lectorat. Selon Laurence Olivier-Messonnier:

L'absence de sang, de mort, de violence armée n'occulte pas les souffrances des Français en temps de guerre. Les jeunes lectrices ne sont pas dupes et ce serait une erreur de leur donner l'illusion que Bécassine vit en dehors des préoccupations matérielles et dans un monde onirique. Sous des dehors plaisants, la gravité alterne avec la satire. En effet, si les albums ne sont guère belliqueux, ils n'en retracent pas moins des traits réalistes et parfois cruels de la guerre. [...] Cependant, malgré les apparences, la visée de l'auteur et de l'illustrateur est contre propagandiste: ils utilisent la caricature pour mieux fustiger les dangers de la propagande, les ridicules d'une population abêtie par l'acculturation guerrière. (60-62)

De plus, si la violence n'y est pas retranscrite en images, elle l'est tout de même par le texte qui est quant à lui, très explicite : «Tiens, sale Boche ! Voilà pour toi sale Boche ! En veux-tu encore sale Boche!»(Bécassine chez les Alliés, 5), crie-t-elle en frappant sur des coussins, une manière somme toute très efficace pour insinuer la haine de l'ennemi dans l'esprit des plus jeunes.

\section{Conclusion}

Que retenir alors, de l'impact des productions illustrées pour la jeunesse et du motif des atrocités allemandes sur l'état d'esprit des petits Français ? A la fois désigné par les adultes comme victime et moteur de la guerre, l'enfant en 1914-1918 fut investi d'un double rôle bien trop difficile à assumer - l'exemple de Dolto était sur ce point éclairant. Déjà entretenu dans une exaltation guerrière et patriotique véhiculée par son apprentissage scolaire, l'enfant doit assurément se reconnaître dans les jeunes héros qui combattent l'ennemi barbare à travers les illustrations de ses journaux préférés. Chez lui et pourtant loin du front, l'enfant s'imagine alors prendre les armes, défenseur héroïque de la Nation. L'objectif visé par les auteurs et illustrateurs de ces récits est atteint, car ils le savent assurément : on ne joue pas innocemment à la guerre.

\section{Bibliographie}

Audoin-Rouzeau, Stéphane. La guerre des enfants : 1914-1918. Paris : Colin, 2004.

Becker, Annette. Les cicatrices rouges : 14-18, France et Belgique occupées. Paris : Fayard, 2010. 
Bettelheim, Bruno. Psychanalyse des contes de fées. Paris : Laffont, 1976.

Bihour Thaïs, «L'esthétisation su viol dans les représentations d'atrocités allemandes (1914 - 1918). Iconographie au service exclusif de la propagande ou expression d'une 'conscience collective'?» Revue TraverSCE, Hors-série consacré à l'année 1914 (automne 2015). 72-84.

Caumery et Pinchon, Joseph-Porphyre, Bécassine pendant la guerre. Gautier-Languereau, 1915.

---. Bécassine chez les Alliés. Gautier-Languereau, 1917.

Docquois, Georges. La chair innocente, l'enfant du viol boche. Paris : Michel, 1918.

Dolto, Françoise. Enfances. Paris : Seuil, 1986.

Gervereau, Laurent. Montrer la guerre ? : Information ou propagande. Paris : SCÉRÉNCNDP : Isthme, 2006.

Histoire du Petit chaperon rouge, racontée par Charles Moreau-Vauthier, illustrée par Guy Arnoux, 1917.

« L'éducation par l'image ». La revue pédagogique, mars 1915.

Olivier-Messonnier, Laurence. «Guerre et littérature de jeunesse, 1913-1919 : analyse des dérives patriotiques dans les périodiques pour enfants ». Thèse de doctorat, Littérature française. Université Clermont-Ferrand 2, 2008.

Ozouf, Jacques et Mona. «Le thème du Patriotisme dans les manuels primaires ». Le mouvement social (oct-déc 1964).

Peureux, Christian. «La propagande à l'école de 1914 à 1918 ». Mémoire de maîtrise, Histoire. Université Panthéon Sorbonne, 1972.

Prévost-Bault, Marie-Pascale. «Le service des enfants, les 'graines de poilus ». Combats de femmes 1914-1918. Dir. Evelyne Morin-Rotureau. Paris : Autrement, « Mémoires/Histoire », 2004.

\footnotetext{
NOTES

${ }^{1}$ Il nous faut établir quelques précisions concernant le terme d'atrocités allemandes : en effet, l'expression se présente parfois avec des guillemets qu'il convient d'utiliser lorsque l'on parle des exactions non avérées et qui ont constitué durant la Grande Guerre, des mythes bien difficiles à démentir - à l'instar du mythe des enfants aux mains coupées. En revanche, lorsque nous parlons des exactions réelles et corroborées par des témoignages, il convient d'ôter les guillemets. Malheureusement, après le conflit, ce terme a posé de nombreux soucis alors que certains historiens mettaient en doute la véracité de certains témoins de ces crimes de guerre, jetant ainsi l'opprobre sur les victimes, et plus particulièrement sur les victimes de viols. Tout amalgame est donc à éviter soigneusement.

${ }^{2}$ En témoignent les nombreux sacrifices que l'on demande aux enfants pour soutenir l'effort de guerre - comme par exemple, faire attention aux quantités de nourriture que l'on mange ou participer à leur manière en cousant des vêtements pour les Poilus qui se battent au front.
} 


\footnotetext{
${ }^{3}$ Intitulé le «Jeu du Pas de l'Oie renouvelé des Boches » et paru entre 1915 et 1917, ce plateau permettait notamment de faire allusion de manière satirique à la démarche militaire allemande, souvent comparée par les caricaturistes de l'époque, au déplacement d'une oie.

${ }^{4}$ Voici ce qu'écrit Christian Peureux à ce propos : «[...] il semble plus grave, que devant ses camarades, on demande à une élève de témoigner sur ce qu'elle a vécu; c'est pourtant ce que rapporte l'Inspecteur d'Académie du Finistère, en le donnant comme exemple pour une leçon de morale : «...Une jeune réfugiée de Malines a pris place sur les bancs de l'école du village : on lui demande de faire le récit des scènes d'atrocités auxquelles elle a assisté, des souffrances endurées ». (50)

${ }^{5}$ Les trois couleurs est un journal pour enfants, publié par la maison d'édition Nilsson à Paris. Il paraît de décembre 1914 à décembre 1919. Tous les numéros sont consultables dans leur intégralité sur le site internet de la Cité Internationale de la Bande Dessinée et de l'Image.

${ }^{6}$ La jeune France. Histoire illustrée de la guerre 1914-1915 - puis La jeune France. Histoire illustrée de la guerre 1914-1916 et enfin La jeune France - est un journal pour enfants qui paraît de 1915 à 1918. Tous les numéros sont consultables dans leur intégralité sur le site internet Gallica.bnf.fr.

${ }^{7}$ Là où Les trois couleurs n'hésitent pas à montrer un soldat allemand faisant tournoyer un enfant dans les airs devant sa famille terrorisée (L'ennemi barbare : «la patrouille allemande passe », parue dans Les trois couleurs 3 (24 décembre 1914). Un autre journal, L'étoile noëliste, présente la vignette d'un bébé cloué à une porte par la soldatesque (s.d.).

${ }^{8}$ La valeur n'attend pas le nombre des années, parue dans La jeune France 18 (11 juillet 1915).

${ }^{9}$ Emile Desjardin, héro de quatorze ans, parue dans La jeune France 19 (18 juillet 1915).

${ }^{10}$ On trouve par exemple la vignette Comment un enfant de douze ans roula les Boches, parue dans Les trois couleurs 8 (28 janvier 1915).

${ }^{11}$ Une bonne idée, parue dans Les trois couleurs 19 (15 avril 1915).
} 